\title{
Research on Characteristics and Programming of Simulated Human Intelligence
}

\author{
Hongwen Cheng \\ Department of Medicine, The Third People's Hospital of Zhongxiang City, Zhongxiang, China \\ Email: chenghwn@aliyun.com
}

How to cite this paper: Cheng, H.W. (2021) Research on Characteristics and Programming of Simulated Human Intelligence. Open Access Library Journal, 8: e7980. https://doi.org/10.4236/oalib.1107980

Received: September 18, 2021

Accepted: October 18, 2021

Published: October 21, 2021

Copyright () 2021 by author(s) and Open Access Library Inc.

This work is licensed under the Creative Commons Attribution International License (CC BY 4.0).

http://creativecommons.org/licenses/by/4.0/

\begin{abstract}
This paper concluded that parallel storage similar to the human brain must have the following characteristics: 1) The connection mode of its information storage is scattered and parallel. 2) The operating pattern of this system must be similar to that of the nervous system for memory, facilitation, and recall. Because of this similarity, we can "borrow" some concepts from neuroscience to describe Advanced intelligent basic programming the attributes of parallel storage systems. 3) The excitation mode of this system must be selective excitation. 4) Memory interference is one of the most important characteristics of parallel storage system. We can't eliminate recall interference completely, but there are a number of steps can take to keep within the limits of our tolerance. 5) Memory interference requires only excitatory memory columns in the contact area. 6) The "memory excitation" mode of the parallel storage system for information must be the fuzzy "memory excitation", which is inevitable of memory interference. Whereafter, This paper describes and discusses how and why to program on the theoretical basis of the first part. Includes an introduction to functions that enable memory, recall, stimulation, excitement. And the relationship between memory, memory, fuzzy excitement, excitement, reward and punishment, attention distribution and their significance in solving memory interference.
\end{abstract}

\section{Subject Areas}

Artificial Intelligence

\section{Keywords}

The Intelligence That Simulates Humans, Parallel Storage, Recall Interference, Attention Distribution, Excited, Rewards and Punishments 


\section{1. 引言}

获得能像人脑一样进行思考的智能软件一直是人工智能界的理想, 而并 行存储、并行计算是人脑思考必备的基础功能。如何用计算机实现与人脑相 似的并行存储、并行计算是本文的目的之一。

人脑进行并行存储与计算的结构基础是它的并行存储结构。科学界对与 人脑类似的并行存储结构研究甚少, 更谈不上通过编程获得这样的结构来应 用。人工智能理论要突破, 必须在并行存储、并行计算、模糊计算兴奋等理 论上有所突破。本文讨论了与人脑类似的并行存储有什么特点与要求。然后 利用这些特点与要求, 通过编程获得了与人脑类似的简单的并行存储结构, 并在此基础上向大家展示了如何通过编程获得并行计算、兴奋、记忆、回忆、 奖惩、注意力的分配等功能。我在《奖惩与学习》 [1]、《注意力问题的系统 讨论》[2]这两篇文章中指出了奖惩通过影响控制注意力的分配, 注意力通过 控制影响对中枢皮质的易化抑制来影响中枢皮质的兴奋。而我在此基础上, 通过编程向大家展示了, 奖惩如何按一定规则影响控制注意力的分配, 注意 力再如何按一定的规则, 易化或抑制 “皮质中枢” 相应记忆柱, 来影响 “中 枢皮质” 的选择兴奋(即通过影响记忆柱的易化兴奋能力, 来影响 “联络区记 忆柱” 的兴奋, 进一步影响主注意对象的选择, 从而影响回忆起的内容), 而 实现思想行为的选择(神经网络的选择兴奋)。《认知对思想行为的影响》 [3] 讨论了人或者智能机器人如何在上面的结构功能基础上获得认知与改造自然 的能力。

这是一种相比于传统人工智能编程理论, 更先进的编写人工智能软件的 理论方法。本理论的一系列设定(如: 记忆的基本单位所对应的结构之间只有 兴奋性 “纤维” 联系, 皮质垂直柱的模拟, 展示框的设定等等)使并行存储、 并行计算能够很好的实现思维的记忆回忆, 从而使人类通过编程获得正真具 有像人脑那样具有高级智能的智能软件成为可能。

通过对并行存储与并行计算的讨论得出, 要使并行存储与并行计算实用 化的最大挑战就是回忆干扰的问题。为解决回忆干扰的问题, 我提出了一系 列的方法, 并在编程中具体应用了这些方法, 部分解决了回忆干扰的问题。

本文的讨论部分参考了人脑的存储结构、存储结构之间的联系特点及人 脑的一些功能特点。可以说, 我们所知道的人脑神经系统的一些结构功能特 点是本文进行讨论的 “指路明灯”, 同时本文的一些讨论结果也有利于我们 对人脑的结构功能的认识。

\section{2. 与人脑类似的并行存储结构, 必然会具有的一些结构与功能 特点}

人脑是个并行存储系统, 它具有强大的功能, 而并行存储结构、并行计 算是这些功能的基础。人脑的并行存储系统有什么特点? 我们该如何模拟 它? 下面先以人脑视觉中枢为例来进行讨论。

休伯尔 [4]和维泽尔发现, 在初级视皮层 (17 区)有一块 1 毫米 $\times 1$ 毫米的 区域, 其中所有神经细胞的感受野都集中在视觉空间的某个区域里, 并且它 
们相邻细胞的最优朝向在 $0^{\circ}$ 到 $180^{\circ}$ 的范围内连续地作有规则的变化。而在厚 度为 2 毫米的垂直范围内, 每个细胞的最优朝向都是一样的, 他们称之为 “朝 向功能柱”。以猴为例, 其 17 区中, 每个柱的宽度约为……每个柱有它自 己的方位轴, 相邻两个柱的方位轴之间, 大约相差 10 度。视野中每个完整的 感受野在视皮质大约占有 2 到 3 毫米的区域，感受野中呈任何角度的东西， 都有方位轴与之相适应的视皮质细胞柱。另外试验证明对感受野刺激强的信 息是图形的边缘。这说明了，一般情况下，一个图形落在视野中，刺激兴奋 强的应是与图形边缘对应的感受野中的一系列柱状结构, 通过刺激兴奋, 这 个图形的信息就 “可能” 对应分散存储在这一系列及随后一系列柱状结构中 (这些兴奋最强的柱状结构, 应该会再兴奋相应的柱状结构)。参考视觉刺激 及其可能的存储特点，我把一个对象的组成信息，分散存储在一组对应的并 行结构中定义为并行存储。

\section{1. 理想化的并行存储结构}

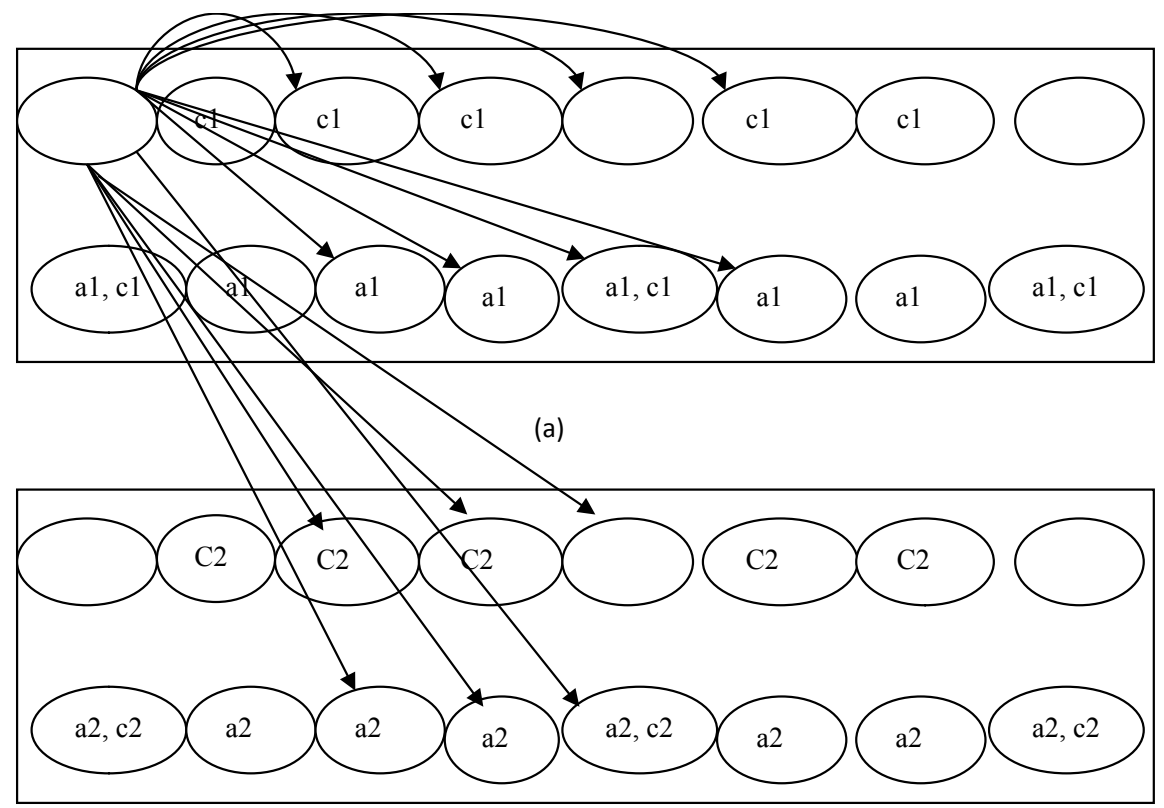

(b)

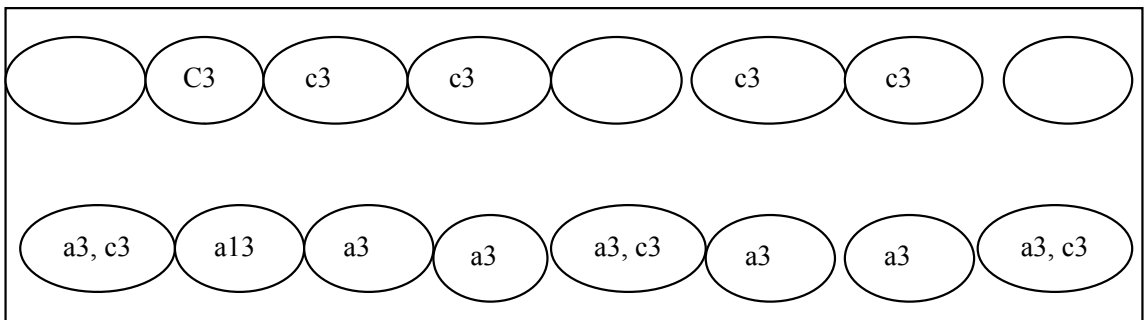

(c)

Figure 1. (a) The parallel structure storage g1; (b) The parallel structure storage g2; (c) The parallel structure storage g3

图 1. (a) 并行存储结构 g1；(b) 并行存储结构 g2；(c) 并行存储结构 g3 
借鉴视觉中枢并行刺激及可能的存储特点, 经过简化, 我构建了理想化 的并行存储结构。顺序存储的特点是, 不同信息顺序存储在一个存储空间的 不同子空间中, 不同信息占用不同的子空间。而本文所指并行存储的特点是, 不同信息并行分散存储在一个共同的存储空间的多个子空间中，不同信息占 用的子空间往往可能有重叠。举例并行存储: 如图 1(a)-(c)分别代表并行存储 结构 $g 1 、 g 2 、 g 3$, 分别存储在它们之中的并行信息是 a1、a2、a3、c1、c2、 c3。并行存储结构 $g 1$ 由 2 行 8 列共 16 个存储单元组成, 这 16 个存储单元中 的每一个单元对应一个基本存储结构(我在下面的编程讨论中, 用基本记忆柱 群来代表它)。而存储的每一个信息都由 8 个 1 或者 2 的数字组成, 每个数字 按顺序分别存储在对应列的相应行中, 比如并行信息结构 $\mathrm{cl}$ ( $\mathrm{c} 1$ 与信息 21112112 对应), 这个信息的第一个数字 2, 存储在并行存储结构的第一列的 第二行中, 第二个数字 1 , 存储在第二列的第一行中......而并行信息结构 a1 (a1 与信息 22222222 对应), 这个信息的第一个数字 2, 存储在第一列的第二 行中, 第二个数字二, 存储在第二列的第二行中 $\cdots \cdots .$. 并行信息结构 $\mathrm{c} 1 、 \mathrm{a} 1 \cdots \cdots$ 分别对应的结构就是某个原始记忆柱群(在下面的编程讨论中要应用这个名 词)。

(图中的箭头表示一个并行存储单元的传出联系所指向的其它存储单元, 如果用基本记忆柱群表示这些存储单元, 就是一个基本记忆柱群的传出纤维 的分布特点, 箭头指向的基本记忆柱群是传出纤维联系分布的基本记忆柱群。 我在下文的编程说明会进一步的讨论)。

\section{2. 并行存储结构的信息传入}

各种信息传入并行存储结构的原则是：由于计算机存储量及计算能力的 限制, 传入的信息必须是信息量较少的并行存储信息。但一个或者多个这样 的信息组合在一起必须能够特异性的表证一个对象。

根据以上的认识并结合人脑视觉中枢的一些特点, 下面以部分模拟视觉 中枢的特点为例来讨论外界刺激信息如何传入并行存储结构(对视觉信息的 处理可能还有其它更好的方法, 但其传入与处理的基本原则应不变)。
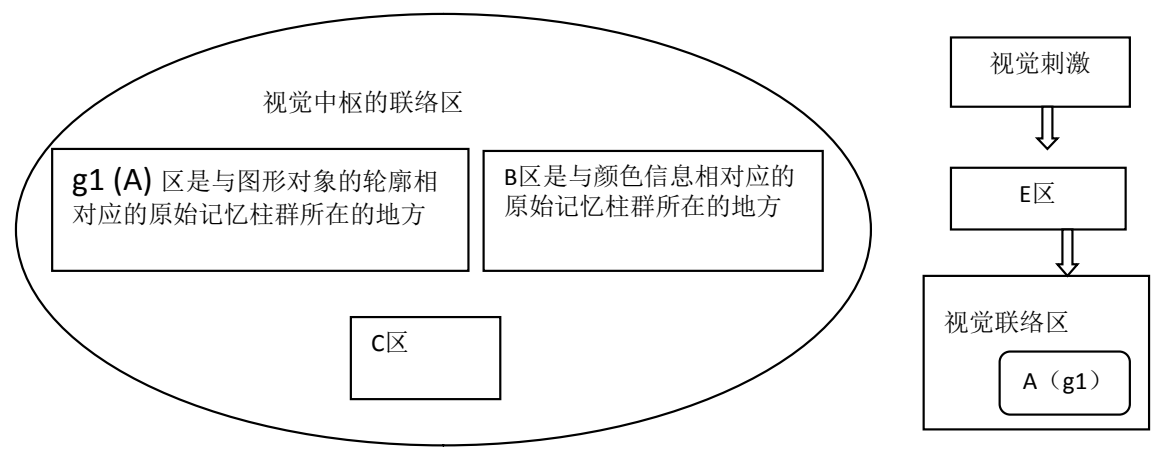

Figure 2. Visual information spreading, processing

图 2. 视觉信息的传入与处理

“在本文, 记忆柱 (具体的定义与特点讨论见下文编程讨论部分) 是智能 
软件兴奋的基本单位的。基本记忆柱群是记忆的基本功能单位所对应的一群 记忆柱。原始记忆柱群是指一个注意对象的一次刺激所对应兴奋的联络区的 记忆柱, 它由一群基本记忆柱群组成。”

如图 2: 假设 $\mathrm{E}$ 区是与图形刺激相对应的地方。当智能软件注意一个对 象时, 不管这个对象在空间的那个位置, 所兴奋的 $\mathrm{E}$ 区的原始记忆柱群都相 同(同时, 对象的空间位置等参数可转化为相应的刺激, 兴奋非 $\mathrm{E}$ 区的相应记 忆柱群)。A 区(用 $\mathrm{g} 1$ 表示)是视觉中枢的联络区的一个亚区, 它与对象的轮廓 信息相对应。

我们可以这样来设置 $\mathrm{E}$ 区的记忆柱的分布与传入信息的关系, 以及 $\mathrm{A}$ 区 的记忆柱的分布与 $\mathrm{E}$ 区传入信息的关系。(为了论述的方便, 本文假设任一个 注意对象的轮廓在 $\mathrm{g} 1$ 区只有 8 个基本存储结构与之对应)。

1) 将 $\mathrm{E}$ 细分成有限的一些区域, 任一个区域都与一次有意义视觉传入的 最小单位(是相对最小, 关键是要能表证对象)相对应(任何一个区域都包含一 群记忆柱), 把这些区域称为最小反应区。将注意对象所充满的空间进行平面 划分, 它所划分的区域与 $\mathrm{E}$ 的最小反应区相对应。这些区域的任何一个区域 的视觉信息在传入到 $\mathrm{E}$ 之前都转化为具有一定角度的线段信息或非线段的其 它信息, 注意对象由这些具有一定角度的线段连接起来构画出其轮廓。与最 小反应区对应的任何一个具有一定角度的线段在 $\mathrm{E}$ 的相应的最小反应区中只 能兴奋一群基本记忆柱群中的一个相应的基本记忆柱群(这群基本记忆柱群 分别对不同的线段信息进行反应, 一次只能有其中的一个基本记忆柱群兴 奋)。

2) 将 $\mathrm{E}$ 区对象的轮廓按一定标准进行处理, 转化成仅由 8 个具有先后顺 序且有一定角度的线段组成的对象，使它们与 $\mathrm{g} 1$ 区的 8 个基本存储结构分别 对应。比如轮廓的第六个线段只能兴奋 $\mathrm{g} 1$ 区的第六行中的相应列的基本存储 结构(基本记忆柱群)。

3) 当然我们也可以用专门的程序或者设备来代替 $\mathrm{E}$ 区的功能, 直接将视 觉刺激信息转换为 “适当的” 并行存储信息传入 $\mathrm{g} 1$ 区 (A 区)。

4) 这样表证一个对象, 必然是模糊表证(因为在不同的环境及时间点下 用有限的信息表证一个可能存在一定变化的对象时, 很难做到完全一致)。

\section{3. 并行存储(记忆)、并行回忆}

A 区的每个最小反应区如果所区分的线段的最小角度是 20 , 则一个最小 反应区能反应不同的角度信息有 $360 / 20=18$, 最小反应区的每个角度信息对 应一个基本记忆柱群, 也就是说一个最小反应区可有 18 个基本记忆柱群, 这 样对应的 $\mathrm{g} 1$ 区每列有 18 个基本记忆柱群(为了编程方便, 本文进行了简化, 一个最小反应区只包含 2 个基本记忆柱群, 分别对应 1 和 2)。一般情况下, 最小反应区不能同时有两个基本记忆柱群强烈兴奋。因而, 当一个刺激对象 引起 $\mathrm{g} 1$ 的反应时, g1 只能有 8 个基本记忆柱群同时强烈兴奋(为什么用 “强 烈兴奋” 来对应信息的刺激与兴奋回忆, 我在后面的选择性兴奋的讨论中会 有答案), 兴奋了的基本记忆柱群相互之间能建立起兴奋性记忆联系, 从而进 行了信息的存储。再进行回忆时, 这 8 个基本记忆柱群通过兴奋性记忆联系 
又能同时兴奋。记忆时的信息被同时存储在不同的基本记忆柱群中，回忆时 又能被同时提取, 这样的记忆、回忆便是并行存储(记忆)、并行回忆。

\section{4. 并行存储运行、计算模式及并行存储信息对应结构之间必然的 联系特点}

我们进行信息的并行存储是为了, 在需要时获得有用的并行存储信息来 应用。获取并行存储信息的一种重要方式, 这也是高级智能所必须具有的： 获得一个并行存储信息后, 再通过这个并行存储信息获得另一个并行存储信 息(就如人脑的兴奋回忆: 回忆起一个记忆内容, 再通过这个记忆内容回忆起 另一个记忆内容) (这一方式是自主思想与行为的基础)。

下面, 通过讨论并行存储系统需要具有哪些条件, 才能够顺利通过一个 并行存储信息获得另一个并行存储信息，来获得实现并行存储所需要的条件。

对于并行存储来说，提取的信息应该是存储的信息(已经有试验证据证明 [5], 我们回忆兴奋的神经网络是记忆时兴奋的神经网络)。通过前文对并行存 储的结构及表示特点的讨论可知，一个并行存储信息被显现提取，它的空间 位置信息(包括组成成分的空间位置信息)也必然直接或间接显现。

这样, 要通过一个并行存储结构 (g1)中的一个并行存储信息(对应的结构 是 a1)获得另一个并行存储结构 (g2)中的并行存储信息(对应的结构是 a2), 这 两个并行存储信息之间就必须建立有联系。这种联系必然包括: 通过 a1 能够 直接或间接获得 a 2 的空间位置信息。也就是说联系建立后 a1 就会直接或间 接的记录了 a 2 的空间位置信息, 包括组成 $\mathrm{a} 2$ 的八组信息的空间位置。否则 通过 a1 便无法定位 a2 而使它显现。

要达成这种联系, 就需要 a1 与 a2 先建立联系, 然后在需要时获取 a1, 再通过 a1 获取 a2。

a1 与 $\mathrm{a} 2$ 的联系关系讨论。我们可以换一种描述方法, 当 $\mathrm{a} 1$ 与 $\mathrm{a} 2$ 的联系 建立的时候, a1 直接或间接记录了 a 2 的空间位置, 通过记录的空间位置信息 a1 能使 a 2 显现, 这相当于 a1 与 a 2 建立了 “兴奋性记忆” 联系(a1 能使 a2 显 现)。然后, 当 $\mathrm{a} 1$ 被选择 “兴奋” (显现)后, 通过 “记忆” 的空间位置信息, 使与 a1 有联系的 a $2 、 c 2 \cdots \cdots$ 相关的信息被显现或者更容易被显现( “易化兴 奋”了与这些信息相应的结构), 从而获得 $\mathrm{a} 2$ 。

通过上面的描述, 可以看出 $\mathrm{a} 1$ 与 $\mathrm{a} 2$ 建立联系后, 再通过 $\mathrm{a} 1$ 获取 $\mathrm{a} 2$ 的 过程, 与神经系统中两个 “刺激信息” 建立兴奋性记忆联系后, 再通过与一 个 “刺激信息” 对应的神经网络易化兴奋一些神经网络, 并从这些易化兴奋 的神经网络中获取与另一个 “刺激信息” 对应的神经网络的过程与特点相似 (一个神经网络 “a 1 ” 直接或间接记录下另一个神经网络 “a2” 的空间位置信 息, 回忆时 “a1” 先兴奋, “激活” 记录的空间位置信息, 再通过记录的空 间位置信息易化其它的神经网络使它们显现, 然后从这些被易化的神经网络 中获取目的神经网络)。这也好理解, 因为本文讨论的并行存储系统就是参照 人脑建立起来的。

因而, 我们可以得出这样的结论: 我们要建立类似于人脑的并行存储系 统, 并使它总能够较好的通过存储的一个并行信息获得另一个与它建立有联 
系的并行信息, 这个系统的运行模式必然与人脑神经系统的记忆、易化兴奋、 回忆特点具有一定的相似性。由于存在这种相似性, 为了方便下面的讨论, 我就直接借用了神经科学的一些名词, 而且, 对于智能软件的编程, 也非常 多的参照了人脑的结构功能特点。

我们建立并行存储系统, 是为了广泛的应用它, 这就需要大量的并行存 储信息之间建立联系, 这样 a1 就应该有与存储在 g2 中的众多信息建立直接 或者间接联系的能力(就如人脑的记忆)。那么对 a1 来说, g2 中的众多并行存 储信息的空间位置信息存储(并行存储信息都存储在相应的空间结构中，只有 定位这些空间结构, 才能获取它们存储的信息)在哪里? 如果存储在另外的结 构中, 这个结构还要与 a 2 及 a 1 再建立联系, 增加了存储结构及联系步骤, 不管是顺序存储还是并行存储, 都不如这些信息存储在 $\mathrm{a} 1 、 \mathrm{a} 2$ 中效率高。人 脑就是这样存储信息的。

并行存储最大的特点是, 经常存在存储信息的结构重叠。所谓存储信息 的结构重叠是指存储在同一个存储结构中的信息的组成结构存在共同部分。 比如存储在 $\mathrm{g} 1$ 中的并行存储信息 21112112 与 22222222 , 结构重叠部分是第 一、第五、第八个数据, 它们都对应存储在 $\mathrm{g} 1$ 的第二行结构中。

如果代表 g2 中的并行存储信息的空间结构信息集中存储在 a1 的某一结 构中, 会造成这些结构要么存储任务过重, 使这些结构过于复杂化, 更难编 程实现, 要么, 信息提取的干扰超过我们忍受的范围。比如：记录的空间信 息全部存储在 $\mathrm{a} 1$ 的第一列中, 那么 $\mathrm{g} 1$ 中存储的数据信息所对应的结构, 只 要其第一列所在空间位置与 a1 相同, 通过这些数据信息获得 $\mathrm{g} 2$ 中的数据时, 所获取的数据都相同(就如人脑中神经网络的兴奋)。也就是说, 由于第一列 结构相同, 而空间位置信息集中存储在第一列, 因而, 它们应该与通过 a1 获 得的数据相同(因为第一个存储结构相同, 会带来即使 g1 中 21112112、 22222222、 $21111111 \cdots \cdots$. 等不同的并行存储数据, 分别与 g2 中不同的并行存 储数据建立了 “记忆” 联系，但通过 21112112、22222222、21111111……等 不同的并行存储数据分别进行 “回忆” 时, 在 $\mathrm{g} 2$ 中显现获得的并行存储数据 会完全相同。如果不好理解, 大家可以人脑的记忆柱、神经元为例进行思考, 就很好理解了), 显然是不行的。信息集中存储在并行存储结构的任何一个存 储结构中都与集中存储在它的第一个存储结构中道理相似, 都是不行的。

如果 a1 中的每个存储结构都存储所有其它存储结构的空间信息, 显然效 率太低(试想人脑皮质的每个柱状结构都与其它所有的柱状结构存在大量纤 维联系, 显然是不可能的)。

因而, 这样就需要存储在 a1 中的 g2 中的并行存储结构的空间位置信息 直接或间接分散存储在组成 $\mathrm{a} 1$ 的各个组成结构中。这种存储模式与人脑类似, 也最容易实现。如何分散存储, 我们可以参考人脑的纤维联系特点(也可以看 我下面的编程讨论)。

\section{5. 并行存储运行计算模式}

在 $\mathrm{g} 1$ 中的 $\mathrm{a} 1$ (22222222)与 $\mathrm{cl}$ (21112112)分别与 $\mathrm{g} 2$ 中的 $\mathrm{a} 2$ (22222222), c2 (21112112)建立了联系, 而 a1 (22222222)从没有与 c2 (21112112)建立过联 
系的前提下, 下面具体讨论, 并行存储如何通过 a1 来获得 $\mathrm{g} 2$ 中的 $\mathrm{a} 2$ 。

当要通过 $\mathrm{g} 1$ 中的 $\mathrm{a} 1$ 来获取 $\mathrm{g} 2$ 中的信息时，由于信息是分散存储的，而 a1 与 $\mathrm{c} 1$ 存在结构重叠, 因而 $\mathrm{a} 1$ 除了与 $\mathrm{a} 2$ 存在联系, 还会通过重叠部分与 $\mathrm{c} 2$ 也存在联系(在通过 a 1 来获取 a 2 时, 会获得 a1 记录的空间信息, 这些信息包 括 a1 与 $\mathrm{c} 1$ 重叠的结构直接或间接记录的部分 $\mathrm{c} 2$ 的空间信息), 只是 $\mathrm{a} 1$ 与 $\mathrm{a} 2$ 的联系相对于与 $\mathrm{c} 2$ 的联系更丰富、更强。同理, 由于并行存储, $\mathrm{a} 1$ 不光与曾 经和它建立有联系的对象 (a2)有联系, 也与那些与 a1 没有建立过联系, 但与 a2 共用存储结构的对象存在联系, 只是比与 a2 的联系弱, 也就是说 a1 与 a2 的联系, 相对于那些与 a1 没有建立过联系的众多对象来说, 最强。因而, 要 通过 $\mathrm{a} 1$ 来获得 $\mathrm{g} 2$ 中的 $\mathrm{a} 2$, 比较可行的方法是: 通过 a1 回忆获取目标并行存 储对象时, 一般只有选择获取与 a 1 联系最强的并行存储信息才最有可能是目 的并行存储对象。

通过长期的大量的记忆联系, al 再显现(被选择兴奋)时, 并不会马上知 道那个或者那些并行存储信息与它联系最强(那个信息是需要的), 只有 a1 尽 可能把其直接或者间接存储的 “重要” 的( “重要” 的一般是联系强的)并行 存储信息都显现出来进行选择，才最有可能正确获得与其联系最强的并行存 储信息。如果丢失部分影响选择的重要信息最终可能会影响信息的选择，在 存在海量信息存储与选择的情况下，这样的丢失对正确选择往往可能是 “致 命” 的。人脑的神经系统就是通过轴突传出兴奋信息，易化所有……人脑的 神经系统符合上面的特点, 我们编程时可以模拟纤维、突触的联系特点。

当然在实际应用中，往往不会简单的只由 $\mathrm{a} 1$ 来 “回忆” (往往需要多个 并行存储信息才能特异表征一个对象), 而是由 a 1 与 a 2 或者更多的对象来共 同 “回忆” 起对象 $\mathrm{a} 3$ 。通过上面的讨论可知: 如果只有 $\mathrm{a} 3$, 即与 $\mathrm{a} 1$ 又与 $\mathrm{a} 2$ 存在强的 “记忆” 联系，当通过 a1 与 a2 共同 “回忆” 时，会显现众多的并 行存储对象,一般是 a3 被 “易化” 的最强(只有 a3 被 a1 与 a2 共同强烈易化), 从而使 a 3 被选择。因而, 要通过 a1、a2 来获得 $\mathrm{g} 3$ 中的 a3, 比较可行的方法 是: 通过 $\mathrm{a} 1 、 \mathrm{a} 2$ 共同回忆获取目标并行存储对象时，一般只有选择获取被“易 化兴奋” 的最强的并行存储对象作为 “回忆” 出的目的对象，才最有可能是 所需要的目的并行存储对象。这是并行存储结构的并行存储信息提取时, 最 重要的计算特点之一。这种计算方式应该也与人脑类似，即从众多被易化的 神经网络中, 选择出最易兴奋的神经网络, 使它强烈兴奋, 作为注意对象。

\section{6. 回忆干扰要求记忆的基本单位之间直接的联系只能是兴奋性 联系。也就是说联络区只有兴奋性记忆柱。}

g1 中的 a1 (22222222)能与 g2 中的任意并行存储对象 a2 (22222222)、c2 (21112112)……建立记忆联系, 并且 a1 能通过记忆, 兴奋这些对象, 产生选 择回忆。可以看出, $\mathrm{g} 1$ 的功能与人脑联络区的功能相似。我在这里将具有 $\mathrm{g} 1$ 类似结构功能的结构, 定义为联络区的结构。

由于人脑的基本兴奋单位是皮质柱状结构，因而我将本文讨论的并行存 储的基本兴奋单位定义为记忆柱。

回忆干扰是指, 由于在并行存储系统中存储的众多并行信息所对应的结 
构往往存在重叠, 而使在提取(回忆)并行信息的过程中, 不仅目标结构会显 现(被易化兴奋), 有的非目标结构也会显现(被易化兴奋), 从而影响目标结构 的获取, 这便是回忆干扰。回忆干扰是影响并行存储系统应用的最主要因素。 并行存储系统必须 “相对” 解决回忆干扰的问题, 否则对存储的并行信息的 提取便会出现问题(提取的信息不是我们所需要的信息), 这样的系统其作用 便大打折扣。为了解决回忆干扰的问题我做了许多设定, 其中重要的一条就 是联络区的记忆柱都是兴奋性记忆柱(这种设定基于神经科学的某些研究成 果[5]), 其兴奋平衡通过其它机制来获得, 如注意的影响, 记忆柱的扩布抑制 等等。否则如果广泛存在抑制性记忆柱, 就会放大回忆干扰的影响, 从而使 我们回忆的内容很难与记忆的内容保持一致。

至于人的大脑皮质中是否只有兴奋性记忆柱现在无法给出确定的证据, 但在皮质局部或不太远的相邻皮质之间的柱状结构之间只存在兴奋性关系 [1]。同时, 虽然只有兴奋性柱状结构, 但柱状结构包含了大量的神经元(人脑 皮质), 它们既有兴奋性的也有抑制的, 它们应参与限制了柱状结构的过度兴 奋。

下面讨论为何规定联络区只有兴奋性而无抑制性记忆柱(如果抑制性的 记忆柱的抑制能力弱, 对其它记忆柱的兴奋产生不了多大影响, 不是这里讨 论的对象。当然, 这样的抑制性记忆柱也无多少存在的意义)的原因:

比如: 用 A、B 两群记忆柱的兴奋分别对应两个记忆对象的刺激兴奋, 且除了 A、B 之间建立有强烈的兴奋性记忆联系外, A、B 也与其它对象建立 有弱的记忆联系。A、B 分别包含 A1、A2、A3、A4、B1、B2、B3、B4 几个 记忆柱群, 每个记忆柱群代表一个记忆的基本单位, 如果不存在抑制性记忆 柱, 当 $\mathrm{A}$ 兴奋后通过记忆兴奋 $\mathrm{B}$ 时, $\mathrm{B}$ 兴奋的就是 $\mathrm{A}$ 与 $\mathrm{B}$ 建立记忆联系时兴 奋的记忆柱群。即使由于回忆干扰, 可能只能选择出 B1、B2、B3 来兴奋, 但我们也可以通过一定的方法将它控制在可容忍范围内。但如果存在抑制性 的记忆柱群 A1, 它兴奋后会强烈抑制 B2、B3 的兴奋。这样, A 兴奋后 A1 会对 B 包含的记忆柱 B2、B3 进行强烈抑制, 而可使 B2、B3 不能被选择兴奋, 这时选择出的记忆柱群便不会是 $\mathrm{A}$ 与 $\mathrm{B}$ 建立记忆联系时兴奋的记忆柱群(也就 是说需要被回忆的内容发生了严重缺失)。再由于回忆干扰 B4 也不能被选择 出来, 而更容易超出系统对回忆差错的容忍范围, 就会经常出现回忆错误。 也就是说如果并行存储系统的联络区广泛存在抑制性的记忆柱, 我们将可能 无法解决回忆干扰的问题。

为了对抗回忆干扰, 我们有必要设置, 在并行存储系统的联络区, 代表 基本记忆单位的记忆柱群(原始记忆柱群)之间的联系只有兴奋性联系。而且 在我们能够通过其它方法, 容易的使记忆柱的兴奋平衡的情况下, 在联络区 设置一些抑制性的记忆柱, 我也看不出, 除了会大大增加回忆的差错率及编 程的复杂度外会有什么其它意义。

这便是我规定联络区的记忆柱只有兴奋性的而无抑制性的原因。这也是 为什么我在模拟人类智能时, 以记忆柱作为记忆的基本兴奋单位, 而不是神 经元或者功能模糊的神经网络作为记忆的基本兴奋单位的一个重要的原因。 


\section{7. 并行存储系统与模糊兴奋回忆的关系}

a1、 $c 1$ 与 g2 中大量的并行存储信息建立 “记忆” 联系后, 由于记忆联系 建立时, 记忆的强弱不同及遗忘的原因, 可能会出现以下情况: 如果 a1 (22222222)与 a2 (22222222)的 “记忆” 联系强度远小于 c1 (21112112)与 c2 (21112112)之间的 “记忆” 联系强度, 通过 a 1 进行 “回忆” 时，由于 a1、 c1 存在结构重叠, 而使兴奋的即包含于 $\mathrm{a} 1$ 又包含于 $\mathrm{c} 1$ 中的结构对 $\mathrm{g} 2$ 的兴奋能 力远强于只包含于 $\mathrm{c} 1$ 中的结构, 就有可能 “回忆” 的是 22222112 (是 $\mathrm{a} 2$ 与 c2 中在组成结构的混合), 而不是 22222222 , 便发生了 “回忆” 干扰, 这是我 们不希望看到的结果。但我们可以通过一定的算法减少这种干扰，但由于大 量并行存储所带来的大量信息重叠及遗忘等原因, 我们并不能完全消除这种 干扰。我们需要在这种干扰存在的情况下, “回忆” 内容是有效、有用的。 否则并行存储系统的建立便失去了意义。也就是说, 如果通过 a1 “回忆” 起 a2, 再通过 a2 “回忆” 起 a3, “回忆” 起来 a3 是这次回忆的最终目的, 代 表回忆是有效、有用的。在回忆干扰的情况下, a1 可能 “回忆” 起与 a2 相似 的 22222212 或者 222222 , 再通过 22222212 或者 222222 “回忆” 起 a3, 最终 “回忆” 的结果是 a3, “回忆” 起来 a3 是这次回忆的最终目的, 代表回忆是 有效、有用的。那么通过与 a2 相似的 22222212 或者 222222 回忆起 a3 便是模 糊兴奋回忆。这些情况我在后面的小程序运行结果中会有讨论。

随着并行存储信息之间记忆联系建立的越广泛越频繁, 同时遗忘发生的 越广泛越频繁, 记忆、回忆干扰的发生也越严重, 我们也越无法完全解决回 忆干扰的问题。既然我们无法完全解决回忆干扰, 还不如认同它们的存在, 只要这些干扰在我们的忍受范围内即可。那么模糊兴奋的存在也是必然的了。

传入信息对对象表证的模糊性，也要求并行存储系统的 “兴奋模式” 是 模糊兴奋。

这样, 要使我们部分模拟人脑获得的并行存储系统存在相对广泛的应用 价值, 并行存储系统对信息的 “兴奋、回忆” 必然是模糊 “兴奋、回忆” 。 模糊兴奋、模糊记忆、模糊回忆是并行存储系统的固有属性。

\section{8. 总结}

如果我们要使建立的并行存储系统能够存储大量的并行信息, 并能较好 的通过存储的某一个并行信息(a1)获得另一个与它建立有联系的并行信息 (a2), 同时使它具有广泛的应用价值。就需要这个并行存储系统至少具有下面 的性质: 1) 表征 $\mathrm{a} 2$ 的信息在 $\mathrm{a} 1$ 中的直接或间接存储是分散的并行存储(如果 a2 与 a 1 的联系信息通过联系模式表示出来, 那么 a2 与 a 1 的联系模式是分散 的、并行的联系)。2) 这个系统的运行模式必然与神经系统的记忆、易化兴 奋、回忆特点具有相似性。由于存在这种相似性, 我们在建立并行存储系统 时可以 “借用” 神经科学的某些名词来描述并行存储系统的性质。3) 这个系 统的兴奋模式必然是选择性兴奋。即选择出最易兴奋(被易化的最强, 也是与 回忆 “发起” 对象联系最强的对象)的记忆柱群作为回忆起的对象。4) 回忆 干扰是并行存储系统运行的必然, 也是最重要的特征之一。我们无法完全消 
除回忆干扰, 但可以通过一系列的措施让回忆干扰带来的影响在我们容忍的 范围内。5) 回忆干扰要求联络区只有兴奋性记忆柱。6) 这个并行存储系统对 信息的 “兴奋、回忆” 模式必然是模糊 “兴奋、回忆” , 这是回忆干扰及传 入信息对对象表证的模糊性的必然。

并行存储最主要的问题是回忆干扰的问题, 回忆干扰产生的原因是表证 不同并行存储信息的原始记忆柱群之间往往会包含一些共同的基本记忆柱 群。下面我提供了解决这一问题的一些方法。

1）与并行存储相对应的并行兴奋、记忆回忆必须是模糊兴奋、记忆回忆。

2）并行存储系统一次只能有有限的基本记忆柱群强烈兴奋。

3）记忆迅速遗忘, 只有少量记忆能转化为中长期记忆, 这样在正常的信 息刺激下产生的记忆可使记忆柱之间的兴奋联系密度控制在一定范围内。通 过遗忘能使不同记忆柱的兴奋能力出现差异, 这种差异是并行回忆的基础之

4) 最易兴奋的并行存储对象被选择兴奋。

5）增加基本记忆柱群数目(减少基本存储单位重叠的比例)。

6) 采取状态兴奋的方法(注意力分配, 如 a2 与 a1 共同易化回忆)。

7) 采取选择注意的策略(去除干扰, 选择出目的对象)。

8) 回忆判断机制(判断回忆正确与否)。

\section{3. 如何编程获得并行存储系统}

明确了并行存储具有的一些性质, 我们在编程获得并行存储系统时便有 了明确的方向。

本文以我用 $\mathrm{vc}$ \#编程获得的一些小程序为例, 来向大家介绍我是如何根据 上面的讨论来设计智能软件的并行存储系统的基本结构及如何运行的。

我是以记忆柱群作为存储结构的基本单位的, 每个记忆柱群又由一个易 兴奋的记忆柱和一个不易兴奋的记忆柱组成。记忆柱部分模拟了人脑的皮质 垂直柱的功能, 皮质垂直柱是特殊的神经网络, 每个皮质垂直柱都含有一定 数目的传入、传出轴突, 它即能被兴奋又能记忆兴奋、遗忘兴奋还能传出兴 奋, 它的功能建立在组成它的神经元的功能之上。本文的记忆柱与广义的神 经网络相比, 它是专门为实现各种易化兴奋、记忆、回忆功能而设计, 有明 确、简单的结构与功能, 便于大规模编写与理论思考。

小程序获得的结构分为联络区和非联络区。联络区的功能在上面已经有 讨论。每个联络区的结构见图 1(a)中 $\mathrm{g} 1$ 的结构。每个输入 $\mathrm{g} 1$ 中的并行信息(如 a1), 包含 8 列数字, 每列数字非 1 即 2 , 数字 1 输入相应列的第一行对应结 构单元, 数字 2 输入相应列的第二行对应结构单元。这个对应的结构单元就 是兴奋记忆的基本单位, 它由一个易兴奋的记忆柱和一个不易兴奋的记忆柱 组成, 我称它为基本记忆柱群。其程序代码如下:

$$
\begin{aligned}
& \text { “clength }[1]=8 ; \operatorname{cheight}[1]=2 ; \operatorname{cwidth}[1]=1 ; \operatorname{clabel}[1]=1 ; \operatorname{cq}[1]=1 ; / / g 1 \\
& \text { clength }[2]=8 ; \operatorname{cheight}[2]=2 ; \operatorname{cwidth}[2]=1 ; \operatorname{clabel}[2]=1 ; \operatorname{cq}[2]=1 ; / / g 2 \\
& \operatorname{clength}[3]=8 ; \operatorname{cheight}[3]=2 ; \operatorname{cwidth}[3]=1 ; \operatorname{clabel}[3]=1 ; \operatorname{cq}[3]=1 ; / / g 3 \\
& \operatorname{clength}[4]=8 ; \operatorname{cheight}[4]=2 ; \operatorname{cwidth}[4]=1 ; \operatorname{clabel}[4]=1 ; \operatorname{cq}[4]=1 ; / / g 4 "
\end{aligned}
$$


每个记忆柱由 9600 个 recordnumber (用 $\mathrm{RN}$ 来表示)组成, 每个 $\mathrm{RN}$ 是一 条记录, 它记录的可以是突触联系时间或者突触的长短期兴奋记忆, 也可以 是记忆柱的兴奋时间。

如图 3: 通过编程获得的记忆柱, 主要分为三个功能区，1）接受突触传 入兴奋的功能区。2) 传出兴奋的功能区。3）综合功能区。1 又分为专门记录 突触兴奋时间、短期、长期记忆及突触其它兴奋属性的功能区。 3 又主要分 为两个功能区。共有 6 个功能区, 编程用 Subzrecordnumber 来表示。

Subzrecordnumber 共有 1600 (我这个小程序是 1200 个 recordnumber) 个 recordnumber, 6 个 subzrecordnumber 共有 $1600 \times 6=9600$ 个 recordnumber。 最后 100 个 recordnumber 记录的是高级综合功能。

\begin{tabular}{|c|c|}
\hline 6、高级功能 & \multirow{2}{*}{$\begin{array}{l}\text { 综合区: 主要记录传入纤维 } \\
\text { 记忆柱的兴奋能力及上一次 } \\
\text { 奋的时间。及其它一些综合 } \\
\text { 数。 }\end{array}$} \\
\hline 5、对传入信息的记忆 & \\
\hline 4、记忆柱的传出信息 & $\begin{array}{l}\text { 传出纤维: 记录传出纤维 } \\
\text { 传出到哪一个记忆柱的哪 } \\
\text {-RN。 }\end{array}$ \\
\hline 3、对应突触长期记忆或其它参数 & 接受传入纤维的突触: 记 \\
\hline 2、对应突触短期记忆或其它参数 & $\begin{array}{l}\text { 奋（包括时间、强度及一 } \\
\text { 些统合性的信息）状态。 }\end{array}$ \\
\hline 1、对应突触兴奋记忆时间或其它参数 & \\
\hline
\end{tabular}

Figure 3. Memory column

图 3. 记忆柱

为了实现信息的分散存储, 参考中枢神经系统纤维的联系特点及结合我 的实际情况。我是这样来设计联络区中记忆柱之间的纤维联系的。

1）联络区中每个基本记忆柱群发出两种纤维联系。一种是与这个联络区 中的其它列的基本记忆柱群的纤维联系, 为内部联系。另一种是与其它联络 区中的其它列的基本记忆柱群的纤维联系, 为外部联系。

2）第一种情况, 如图 1(a)、图 1(b), 每个基本记忆柱群发出的纤维分指 向与其位于同一联络区, 并间隔一列的连续排列的四列记忆柱群。第二种情 况, 如图 1(a)、图 1(b), 每个基本记忆柱群发出的纤维分指向与其位于不同 联络区, 并间隔一列的连续排列的三列记忆柱群。

3）这样, 当 a1 与 a 2 建立记忆联系后, a2 的空间信息便通过 $\mathrm{a} 1$ 到 $\mathrm{a} 2$ 的 纤维联系的强化(通过与纤维对应突触的兴奋记忆来实现)得到间接存储。由 于纤维联系是分散分布的, 存储的空间信息也必然是分散的。

部分代码如下:

if (opn !=n)//中枢不相同, 这是外部联系。 
\{

//计算出基本 length, height

for (outputsame $=1$; outputsame $<=$ outputnotsamen; outputsame++)

// outputnotsamen 的值表示基本记忆柱群传出纤维联系的列的数目

\{

oplengthn = clengthn $+1+$ outputsame; //计算第几个传出

if (oplengthn $>$ clength[opn])//计算出 length, 从第几个基本记

忆柱群开始计算

\{

oplengthn = oplengthn-clength [opn];

if $(\mathrm{opn}==\mathrm{n}) / /$ 中枢相同, 内部联系

$\{$ //要明确中枢的位置

......

这些简单设定都是结合了我的实际情况而作出的, 当然如果你的电脑存 储空间足够大, 电脑速度足够快, 并且编程能力足够好, 你也可以把联络区 的列与行都增加多倍, 同时每个记忆柱的 RN 也增加到几万甚至几十万个, 这样在扩展其功能的同时, 也能减少回忆干扰的发生。

\section{4. 顺序计算转换为并行计算}

并行存储对应的必然是并行计算, 而 PC 是顺序计算, 为了用 PC 的顺序 计算来模拟并行计算, 我引入了展示框的概念。展示框是由 $\mathrm{RN}$ 组成的, 它 的作用是记录某一刻(一短时间段)所有被 “兴奋” 的记忆柱, 以使 PC 逐一读 写完这一时刻被兴奋的记忆柱, 并进行一系列操作后, 再逐一读写下一时刻 被兴奋的记忆柱, 通过这种转换就可使一次只能进行一次读写的 PC 机也能 模拟人脑的并行兴奋特点。

下面是程序代码:

下面的的代码定义了展示框 6, 它的数据记录在文件 “d:// xfy.txt” 中,

"FileStream xfy = new FileStream("d:// xfy.txt", FileMode.Create);//6 ......

void fdlabelfun(int fdlabel, ref FileStream fd)

switch (fdlabel)

......

case 6:

$$
\mathrm{fd}=\mathrm{xfy}
$$

break;"

下面的代码是读取与存储数据到展示框 6 中:

“zsk =6;

getfun(labelrecordnumber, ref labelnumber, zsk, fd); 
zsk = 6;

putfun(lingshilabel, xfrecordnumber, zsk, fd);"

下面的代码实现周期性兴奋, 一个兴奋周期就是记忆柱的一个兴奋及传 出兴奋的周期:

getfun(labelrecordnumber, ref jrlabell, zsk, fd);

for (int labelrecordnumberr $=2$; labelrecordnumberr $<=$ jrlabell + 1; labelrecordnumberr++)

$\{\ldots . .$.

\#region

//这一兴奋周期的时间。它是记忆柱总的兴奋时间, 也 是展示框的兴奋时间,

if ((int)label == 1) //确定本兴奋周期读的展示框的标 志(label)。

忆标志

$$
\begin{aligned}
& \text { label = 2; } \\
& \text { qlabel = 1; //后一兴奋周期展示框的标志, 也是记 }
\end{aligned}
$$

int labelrecordnumber $1=1$;

labelrecordnumber $=($ label -1$) * 4000+1$; //本周期被 读取的展示框的记录标志。它记录的是前一兴奋周期记录下来的将要兴奋的 记忆柱;

int qlabelrecordnumber $=($ qlabel -1$) * 4000+1 ; / /$ 后一 兴奋周期将要兴奋的记忆柱。也是前一兴奋周期所在展示框;

\#endregion

\section{5. 软件的运行}

小程序主要分两部分。一部分是建立文件系统，一部分是程序的功能实 现部分。并行存储文件(见附件并行存储文件.txt)中的程序代码是编程建立文 件系统的程序, 点击调试, 程序运行, 会出现运行窗口, 点击 botton 按钮, 就会在 D 盘建立几个文件, 其中 textfil1 文本文件就是以记忆柱为基础建立的 文件系统。

并行 (见附件并行.txt)中的程序代码是程序的功能实现代码。运行程序代 码, 它就会通过 “for (int labelrecordnumberr $=2$; labelrecordnumberr $<=$ jrlabell +1 ; labelrecordnumberr ++$)$ ” 周而复始的运行, 每个周期都是记忆柱的 一个兴奋周期。每个计算周期都会重复以下事件。接受刺激传入、主注意对 象选择、易化兴奋、传出兴奋、奖惩预期、注意力分配等等。这些事件的运 算实现都是围绕展示框进行的。 
整个软件运行的核心目的是存储(记忆)有用或可能有用(有用、需要的对 象会带来奖赏)的对象, 提取(回忆)需要或可能需要的对象, 这些对象在被存 储与提取时就是主注意对象，主注意对象兴奋的过程就是存储与提取信息的 过程。也就是说整个软件运行的核心是围绕主注意对象展开的。

\section{1. 小程序运行的主要机理是}

各个对象所能带来的预期奖惩按一定计算规则决定注意力的分配，注意 力再按一定的计算规则, 决定注意力分配中枢对皮质中枢对应存储结构的易 化抑制强度, 来影响中枢皮质的选择兴奋(即通过影响记忆柱的易化兴奋能 力, 进一步影响主注意对象的选择), 从而影响回忆起的内容。

对象分配的注意力越多, 其受到的易化越强, 越容易被选择成为主注意 对象。而对象分配的注意力的多少的决定因素是奖惩预期, 对象与越强的奖 赏存在密切关系, 其被分配的注意力越多。

下面讨论主注意对象的选择与兴奋。

1) 根据讨论获取的并行存储的特点, 我暂时设计了主注意对象的简单选 择方法。刺激或者回忆时, 按照一定规律, 在一个兴奋最强的中枢的每列中, 选择出兴奋最强且兴奋到一定程度的易兴奋的记忆柱, 组成主注意对象, 主 注意对象对应的基本记忆柱群就是刺激兴奋或者回忆兴奋的内容。兴奋成为 主注意对象的记忆柱群的兴奋能力远大于其他记忆柱群的兴奋能力, 这些记 忆柱之间产生的兴奋性记忆联系也远强于其他记忆柱之间产生的记忆。这是 它们今后成为一个整体被回忆的基础之一。

2) 一主注意对象(a1)兴奋几个兴奋周期后, 组成它的易兴奋性记忆柱兴 奋停止(这时 a 1 便不能再被选择成为主注意对象)。而不易兴奋的记忆柱的兴 奋还持续一段时间, 这些不易兴奋的记忆柱与这时选择成为主注意对象的强 烈兴奋的易兴奋的记忆柱能建立起强烈的兴奋性记忆联系。这是通过一存储 的对象来获取另一个存储对象的基础。

这样当一主注意对象兴奋后, 它的不易兴奋的记忆柱就会通过记忆的兴 奋, 强烈兴奋一些易兴奋的记忆柱, 这些易兴奋的记忆柱也会相互兴奋, 它 们之中曾经同时成为主注意对象的易兴奋的记忆柱相互之间的兴奋能力一般 会显著强于其它易兴奋的记忆柱之间的兴奋能力, 因而它们的兴奋也显著 强……在这一主注意对象的兴奋终止后, 它的易兴奋的记忆柱兴奋也停止, 这时就会从被不易兴奋的记忆柱强烈兴奋的易兴奋记忆柱之中再选择出兴奋 最强的易兴奋的记忆柱对应的原始记忆柱群作为主注意对象。从而开始下一 个兴奋周期。

\section{2. 程序介绍}

这里向大家简单介绍我按照前文的讨论编程的两个小程序。较具体的讨 论见链接内容。

\section{小程序的结构、机理及运行过程、结果}

这两个小程序向大家展示了, 如何编程获得并行存储结构(这种并行存储 结构是简单化、理想化的, 它是部分模拟了人脑的存储结构而获得的。)和其 
基本结构功能单位(记忆柱), 及它们之间如何联系。如何将并行信息存储在 并行存储结构中, 存储的信息之间如何建立记忆联系, 如何进行并行计算, 如何通过刺激或存储的信息易化兴奋相关存储信息, 并通过选择获得有用的 存储信息等等。

程序的运行实现了记忆、回忆、模糊兴奋、奖惩预期、注意力分配等等 功能, 展示了奖惩、注意力分配如何影响记忆柱的兴奋, 并部分解决了回忆 干扰的问题。

对这两个小程序的讨论文章 ${ }^{1}$ 及一些资料 ${ }^{2}$ (包括程序代码及一些程序运 行结果)可通过网盘获取。

\section{6. 高级智能需要与人脑类似的并行存储来参与实现}

为什么我认为顺序存储无法实现高级智能, 而与人脑类似的并行存储能 够实现哪?

首先, 通过对我编程的小程序的讨论, 证明了编程获得的与人脑类似的 并行存储可以实现易化、兴奋、记忆、回忆、奖惩、注意力分配、奖惩预期 等高级智能必须具有的功能。

另外, 考察人脑的记忆回忆联系可知, 在长期的实践过程中, 各个记忆 对象之间能够方便的建立记忆联系, 并相互回忆, 这对高级智能来说是非常 重要的, 比如有了这种能力我们才能轻易的对自己所思考的内容进行评估, 又比如对于一个物体 $(\mathrm{m})$ 的表征, 只用 $\mathrm{m}$ 的一个 “图形属性” 产生的主注意 对象来表征 $\mathrm{m}$, 显然不足以特异性的表征 $\mathrm{m}$, 必须一群特异的 “图形属性” 才能将 $\mathrm{m}$ 与大量的其它物体区分开来。要使这一群特异的 “图形属性” 所对 应的主注意对象发挥识别作用, 就需要它们之间能够方便的建立记忆联系, 并相互回忆。

通过前面的讨论可知, 与人脑类似的并行存储结构的各个存储对象之间 “天然存在直接或间接的纤维联系”，使它们之间能够容易的建立记忆联系 (只要使它们先后或者同时兴奋), 并且不会增加存储空间。而顺序存储的各 个存储对象之间缺乏 “那种天然的联系” ，要使它们之间方便的建立记忆回 忆联系则比较困难, 同时顺序存储建立新的记忆联系时需要增加存储空间, 而在长期的学习过程中, 这种增加必然是超负荷的。

高级智能一个必须具有的重要特点就是, 对某个对象的回忆(比如并行存 储对象 a6), 往往不是通过一个对象孤立的回忆, 而是通过多个对象来实现(通 过 a1、a2、a3 等共同回忆来使 a6 被选择兴奋, a 1 单独与 a6 的联系并不是最 强, 但在 a1、a2、a3 等的共同参与下, a6 受到最强易化。) (比如我们平常所 说的情景记忆回忆, 及前文关于 $\mathrm{m}$ 的区分, 就是这种情况), 这种回忆在通过 每一个对象(a1)进行回忆时并不能知道那些是需要的回忆, 那些是不需要的回 忆, 因而, 需要所有与它们建立过联系的存储对象被易化, 并从这些被易化 的对象中选择出目标对象。并行存储能通过注意力分配中枢轻松高效实现上 面所述过程(因为并行存储要实现上述过程, 虽然需要易化的对象是非常多 1链接: https://pan.baidu.com/s/1-afn6Uhbryreoh9YJFKbQg, 提取码: wwik。

2 链接: https://pan.baidu.com/s/1eLTW9MB_6h_NoZ3vtwtIVQ, 提取码: ugp7。 
的, 但它只需要易化有限的一些基础存储结构就可以实现易化趋于无限的并 行存储对象, 也就是说需要易化的对象是非常有限的), 而顺序存储则如何实 现呢? 很难, 即使强行实现, 效率也低, 缺乏实用价值。

可以看出, 这样编程出来的人工智能软件的存储对象之间能够容易的建 立直接或者间接的联系, 也就是说智能软件在奖惩及注意力分配的限制下, 能够通过直接或者间接的兴奋, 回忆起与它所感知与思考的对象相关的存储 内容(比如一些属性, 一些认知等等), 并通过这些回忆的存储内容对它们产 生认知, 从而意识到它们(正确的认知能带来奖赏)。因而, 从理论上说, 未 来我们可以通过适当的编程, 让这样的人工智能软件具有意识。

由于这个小软件它不断追求更强的奖赏, 并逃避惩罚, 因而它可以有自 主行为。

\section{7. 结论}

通过前文的讨论可知, 与人脑类似的并行存储必然具有以下特点: 1) 其 存储信息的联系模式是分散的、并行的联系。2) 这个系统的运行模式必然与 神经系统的记忆、易化兴奋、回忆特点具有相似性。由于存在这种相似性, 我们可以 “借用” 神经科学的某些名词来描述并行存储系统的性质。3) 这个 系统的兴奋模式必然是选择性兴奋。即选择出最易兴奋(被易化的最强, 也是 与回忆 “发起” 对象联系最强的对象)的记忆柱作为回忆起的对象。4) 回忆 干扰是并行存储系统运行的必然, 也是最重要的特征之一。我们无法完全消 除回忆干扰, 但可以通过一系列的措施让回忆干扰带来的影响在我们容忍的 范围内。5) 回忆干扰要求联络区只有兴奋性记忆柱。6) 这个并行存储系统对 信息的 “回忆兴奋” 模式必然是模糊 “回忆兴奋” , 这是回忆干扰的必然。 要使这样的并行存储结构实用化, 就必须解决回忆干扰的问题。在以上讨论 的基础上, 本文提供了并行存储与计算的基础理论与编程方法, 随后按照这 些理论与方法进行了编程讨论, 证明了这样编程获得的软件可以实现易化、 兴奋、模糊兴奋、记忆、回忆、遗忘、奖惩学习、思想行为的动力预期、注 意力分配等等这些人的高级智能所必须具有的功能。

高级智能需要与人脑类似的并行存储来参与实现。

本文的并行存储与计算是与当前流行的人工智能理论(比如深度学习、机 器学习等等)在基础结构与计算方法上都有所不同的理论, 它更多的模拟了人 脑的存储结构与计算模式, 是一种全新的人工智能编程理论与方法, 它的一 系列的设定实现了并行存储与计算, 使高级智能的编程获得成为可能。

\section{Conflicts of Interest}

The author declares no conflicts of interest.

\section{References}

[1] Cheng, H.W. (2007) Relationship between Reward and Punishment Nerve Centers and Learning. Mind and Computation, 208-212.

[2] Cheng, H.W. (2010) System Discussion of Attention Problems. Mind and Compu- 
tation, 4, 216-227.

[3] 程洪文. 认知对思想行为的影响 [EB/OL]. 中国科技论文在线. http://www.paper.edu.cn/releasepaper/content/201404-304, 2014-04-22.

[4] 乔治.阿德尔曼, 巴里.史密斯, 等. 神经科学百科全书 $[\mathrm{M}]$. 杨雄里, 等, 译. 上海: 上海科学技术出版社, 1992: 528-531.

[5] Polyn, S.M., Natu, V.S., Cohen, J.D., et al. (2005) Category-Specific Cortical Activity Precedes Retrieval During Memory Search. Science, 310, 1963-1966. https://doi.org/10.1126/science.1117645

\section{Appendix (Abstract and Keywords in Chinese)}

\section{拟人智能的特点及编程研究}

摘要: 本文通过讨论得出, 与人脑类似的并行存储必然具有以下特点: 1) 其 存储信息的联系模式是分散的、并行的联系。2) 这个系统的运行模式必然与 神经系统的记忆、易化兴奋、回忆特点具有相似性。由于存在这些相似性, 我们可以 “借用” 神经科学的某些名词来描述并行存储系统的性质。3) 这个 系统的兴奋模式必然是选择性兴奋。4) 回忆干扰是并行存储系统运行的必 然, 也是最重要的特征之一。我们无法完全消除回忆干扰, 但可以通过一系 列的措施让回忆干扰带来的影响在我们容忍的范围内。5) 回忆干扰要求联络 区只有兴奋性记忆柱。6）这个并行存储系统对信息的 “回忆兴奋” 模式必然 是模糊 “回忆兴奋”, 这是回忆干扰的必然。随后, 本文介绍、讨论了如何 在上述结论的基础上进行编程及为什么这样编程。包括编程实现记忆、回忆、 刺激传入、模糊兴奋、兴奋、奖惩、注意力分配等功能, 及兴奋、奖惩、注 意力分配之间的关系以及它们对解决回忆干扰的意义。

关键词: 拟人智能, 并行存储, 回忆干扰, 注意力分配, 兴奋, 奖惩 